\title{
Numerical simulation of diffusion-controlled solid-state reactions in spherical particles
}

\author{
Phillip Gwo-Yan Huang ${ }^{a}$, Chung-Hsin Lu ${ }^{\mathrm{b}}$, Tony Wen-Hann Sheu ${ }^{\mathrm{a}, *}$ \\ ${ }^{a}$ Department of Engineering Science and Ocean Engineering, National Taiwan University, Taipei, Taiwan, ROC \\ ${ }^{\mathrm{b}}$ Department of Chemical Engineering, National Taiwan University, Taipei, Taiwan, ROC
}

Received 9 October 2003; accepted 10 October 2003

\begin{abstract}
The three-dimensional time-dependent diffusion equation, subjected to a chemical reaction at the interface of the reacted layer and unreacted core, used for simulating diffusion-controlled solid-state reactions is solved by a finite volume method in a body fitted curvilinear co-ordinate system. The simulated reaction zone is seen to advance towards the center of a spherical particle. The concentrations of reactants and product are also predicted. Within the reacted zone, the simulated diffusion rates at different spherical cross sections reveal a clear picture of the unsteady diffusion process. The predicted kinetic curves at different conversion constants help to understand the kinetics of diffusion controlled solid-state reactions in spherical particles.
\end{abstract}

(C) 2003 Elsevier B.V. All rights reserved.

Keywords: Diffusion-controlled; Solid-state reactions; Spherical particle; Diffusion rates; Kinetic curve; Conversion constants

\section{Introduction}

Recent advances in computer hardware technology and computer aided engineering (CAE) software technique have made numerical exploration of three-dimensional complex physics feasible [1]. We are, therefore, motivated to numerically explore the solid-state reaction kinetics in spherical particles. Amongst the solid-state reaction models, the diffusion-controlled model [2], the interface-reaction controlled model [3] and the nucleation-controlled model [4-6] are often referred to. In ceramic and metallic processes, the solid-state reaction is the result of an intimate mixing of fine powders. For an isothermal reaction, the diffusion of reactants proceeds a slower rate than those in the other steps. The formation of reacted zone depends, therefore, on the rate of diffusion [7].

In 1927, Jander [2] proposed the diffusion-controlled core-shell model using the planar diffusion assumption to correlate the reacted fraction with time. The three-dimensional diffusion assumption was made later by Ginstling and Brounshtein [8] in their core-shell model.

\footnotetext{
* Corresponding author. Tel.: +886-2-23625470x246; fax: +886-2-23929885.

E-mail address: twhsheu@ntu.edu.tw (T.W.-H. Sheu).
}

More recently, Carter [9] further took into account the molal volume difference of the reactant and product. Under certain assumptions, this refined model can estimate the degree of conversion of the powder reaction by deducing the kinetic equations that relate the reacted fraction with time. However, the steady diffusion assumption made in a finite-thickness reacted layer can limit the applicability and, at the same time, can deteriorate the predicted range of conversion rate.

The objective of the present solid-state reaction study is to develop a numerical model for solving the diffusing equation that subject to an interfacial chemical reaction. The fully conservative finite-volume method is employed to solve the diffusion equation in body-fitted coordinates [10]. This coordinate transformation technique, which has been well accepted to simulate flow problems with irregular geometries, is often employed together within the multi-block grid technique [11] to improve the prediction quality. In this study, we consider an unsteady diffusion in a given thickness of reacted zone. Our aims are to estimate the degree of conversion relative to the full course of reaction and the time-evolving concentrations of reactants and product within the whole particle.

The simulated results include the three-dimensional reaction front that gradually advances towards the center of a 
spherical particle. The simulated diffusion rates at different spherical cross sections in the reacted layer help to understand the diffusion process. To validate the numerical model, the simulated solutions are compared with the data of Ginstling and Brounshtein [8]. Furthermore, the degrees of conversion obtained at different conversion constants are also investigated.

\section{Diffusion-controlled solid-state reaction}

\subsection{Conversion kinetics}

In a spherical particle, the solid-state reaction $\mathrm{A}+\mathrm{B} \rightarrow$ $A B$ is shown in Fig. 1(a) and (b). Initially, the investigated reactant $\mathrm{B}$ is surrounded by the reactant $\mathrm{A}$. After $t>0, \mathrm{~A}$ immediately reacts with $\mathrm{B}$ and produces a product layer $\mathrm{AB}$ in a spherical shell which covers the unreacted core of $B$. In the course of forming $\mathrm{AB}, \mathrm{A}$ diffuses through the product layer $\mathrm{AB}$ and then reaches the unreacted $\mathrm{B}$, followed by $\mathrm{a}$ continuous growth of product layer. During this solid-state reaction, the diffusion speed of $\mathrm{A}$ is normally smaller than the chemical reaction speed. As a result, the diffusion process governs mainly the conversion kinetics. Usually, we call kinetics of conversion the relationship between the reacted fraction and the time elapsed. The degree of conversion, $\alpha$, is defined as

$\alpha=\frac{\mathrm{AB}_{\mathrm{r}}}{\mathrm{AB}_{0}}$

where $A B_{r}$ is the mole (or weight) of the reaction product layer during the conversion process. As for $\mathrm{AB}_{0}$, it denotes the total mole (or weight) of the product in the entire particle at a time when the conversion process is complete.

\subsection{Diffusion equation}

In the diffusion-controlled solid-state reaction, the diffusion process of $\mathrm{A}$ in the product layer $\mathrm{AB}$ can be simulated by solving the time-dependent species transport equation, which needs to be supplemented with the appropriate boundary and initial conditions. In a notation-free coordinate, the unsteady diffusion equation can be expressed as

$\frac{\partial\left(\rho C_{\mathrm{A}}\right)}{\partial t}-\nabla \cdot\left(\rho D \nabla C_{\mathrm{A}}\right)=0$

where $t$ is time, $\rho$ the particle density, $C_{\mathrm{A}}$ the concentration of $\mathrm{A}$, and $D$ the diffusivity of $\mathrm{A}$ in the product layer. Under the constant-coefficient assumption, equation (2) turns out to be the equation known as Fick's second law:

$\frac{\partial C_{\mathrm{A}}}{\partial t}-D \nabla^{2} C_{\mathrm{A}}=0$

As depicted in Fig. 1(a) and (b), the particle is surrounded by reactant $\mathrm{A}$. Therefore, a fixed concentration value for $\mathrm{A}$ is prescribed on the outer surface of the particle. Initially, the particle under the current investigation contains only the reactant $\mathrm{B}$. No reactant $\mathrm{A}$ or product $\mathrm{AB}$ is seen at that moment.

\subsection{Interfacial chemical reaction}

Considering an immediate reaction $\mathrm{A}+\mathrm{B} \rightarrow \mathrm{AB}$, the interfacial concentrations of two reactants $\left(C_{\mathrm{A}}\right.$, and $\left.C_{\mathrm{B}}\right)$ and one product $\left(C_{\mathrm{AB}}\right)$ can be calculated as

$$
\begin{aligned}
& C_{\mathrm{A}}=\max \left(0, C_{\mathrm{A}}^{*}-C_{\mathrm{B}}^{*}\right) \\
& C_{\mathrm{B}}=\max \left(0, C_{\mathrm{B}}^{*}-C_{\mathrm{A}}^{*}\right) \\
& C_{\mathrm{AB}}=C_{\mathrm{AB}}^{*}+\min \left(C_{\mathrm{A}}^{*}, C_{\mathrm{B}}^{*}\right)
\end{aligned}
$$

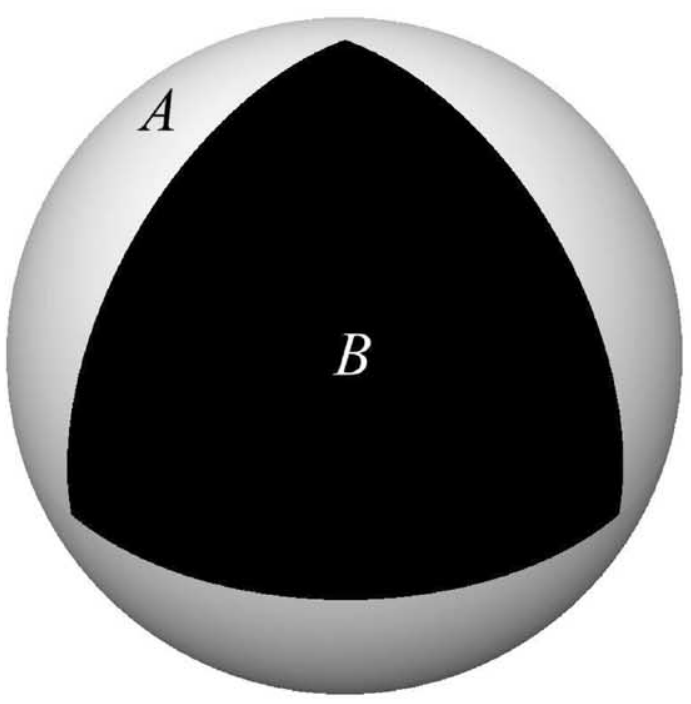

(a)

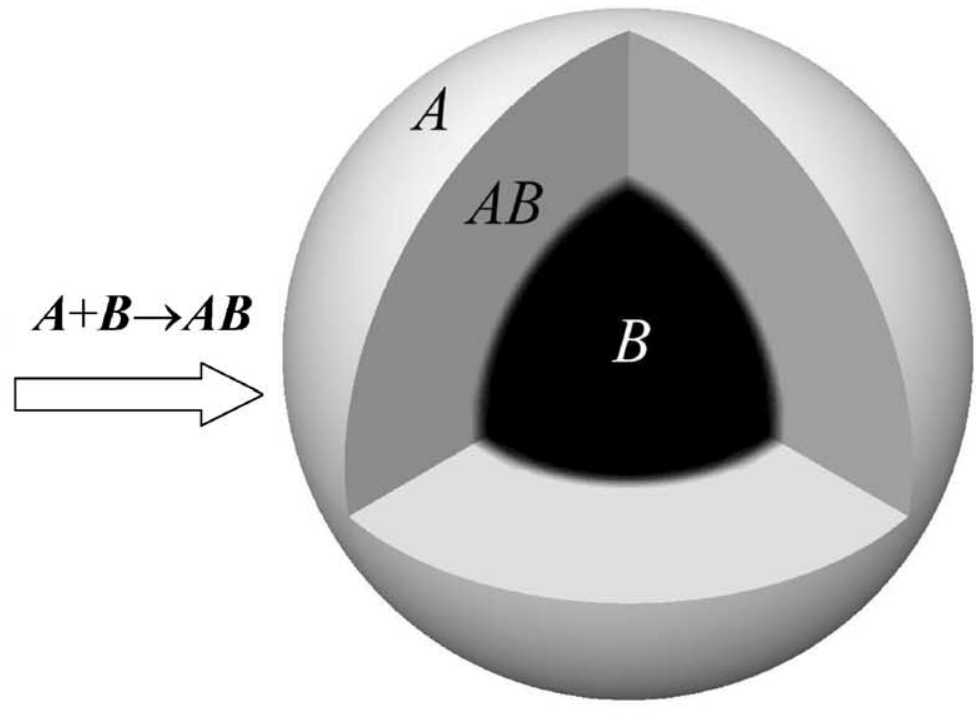

(b)

Fig. 1. A diffusion-controlled solid-state reaction $\mathrm{A}+\mathrm{B} \rightarrow \mathrm{AB}$ : (a) at the initial state; (b) after a reacted layer produced. 
In the above, $C_{\mathrm{A}}^{*}, C_{\mathrm{B}}^{*}$ and $C_{\mathrm{AB}}^{*}$ denote the concentrations of $\mathrm{A}, \mathrm{B}$ and $\mathrm{AB}$, respectively, at the interface before the chemical reaction starts.

\section{Numerical model}

\subsection{Finite volume numerical approach}

The current simulation involves applying a conservative, unstructured, finite-volume code in a body-fitted coordinate system [10]. All variables are stored at the volume center. The equation (3) is integrated over each control volume to obtain a discrete equation that connects the primary variables at the volume center with its neighbors. By integrating the working equation (3) over an arbitrary domain $\Omega$ and applying the Gauss divergence theorem, we have

$\int_{\Omega} \frac{\partial C_{\mathrm{A}}}{\partial t} \mathrm{~d} V-D \int_{\partial \Omega} \nabla C_{\mathrm{A}} \cdot \vec{n} \mathrm{~d} S=0$

In the above equation, $V$ denotes the control volume, $S$ the control surface, and $\vec{n}$ the unit normal vector. All spatial derivative terms are discretized by the second-order centered scheme while a fully implicit backward time stepping scheme [12] for the time derivative term.

Within the geometrically symmetric and physically uniform framework, one octant of the spherical particle, schematic in Fig. 2, is sufficient. The investigated domain was subdivided into four blocks. Within each block, there are hexahedral grids, as shown in Fig. 2(b). To acquire an adequately resolved solution, computations with increasing grid density are performed.

The present numerical simulation starts with application of a commercially available CAD (Computer Aided Design) tool CATIA [13] to create the solid geometry of the spherical particle. Then, the species properties, boundary conditions, initial conditions and chemical reaction conditions are provided. This is followed by using ICEM-HEXA [14] to create the physically meaningful multi-block grids. The finite volume code CFX-4 [15] is then applied to obtain solutions for the working equations. The results obtained at different grid densities are compared for confirming whether or not the grid-independent solutions have been obtained. The predicted growth of product layer is visualized by virtue of a post-processor FIELDVIEW [16]. Finally, we discuss the simulated concentration distributions, diffusion rate through the spherical cross section, degree of conversion and the complete conversion time $t_{\mathrm{c}}$ (when $\alpha=1.0$ ).

\subsection{Model for comparison}

The numerical results are compared with the data obtained from the Ginstling and Brounshtein's model [8]:

$1-\frac{2}{3} \alpha^{\prime}-\left(1-\alpha^{\prime}\right)^{2 / 3}=K t$,

Note that the above kinetic equation was derived under the steady diffusion assumption in a finite-thickness reacted layer. In Eq. (8), $\alpha^{\prime}$ is the degree of conversion, which

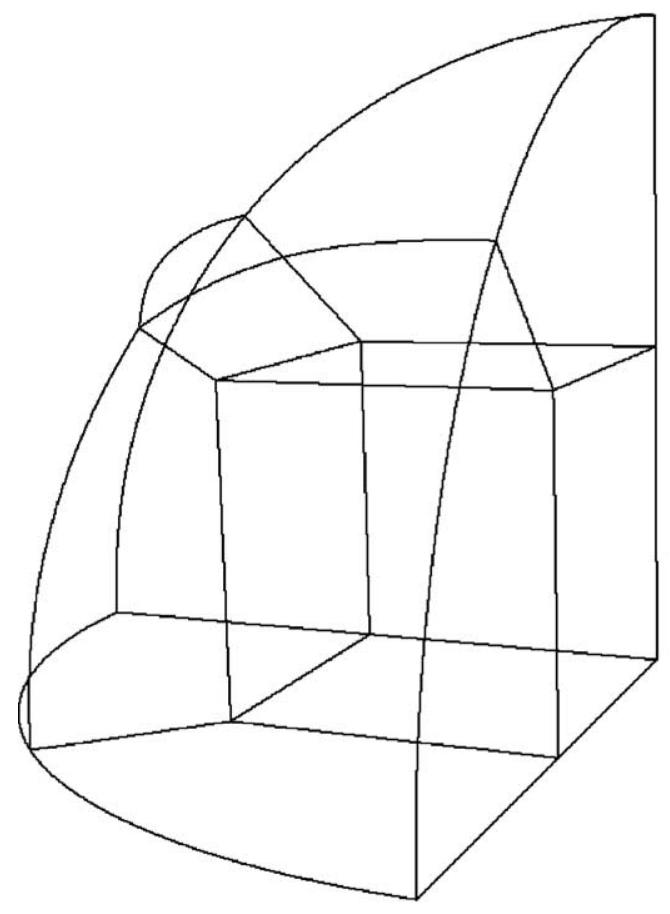

(a)

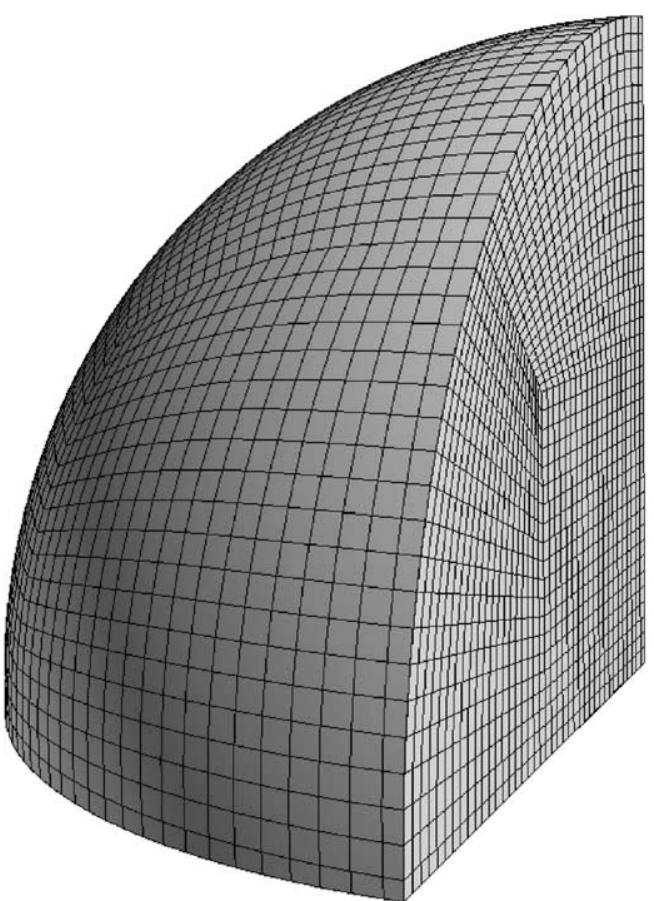

(b)

Fig. 2. Schematic of the computational domain: (a) subdivided multi-blocks; (b) distributed hexahedral grids. 


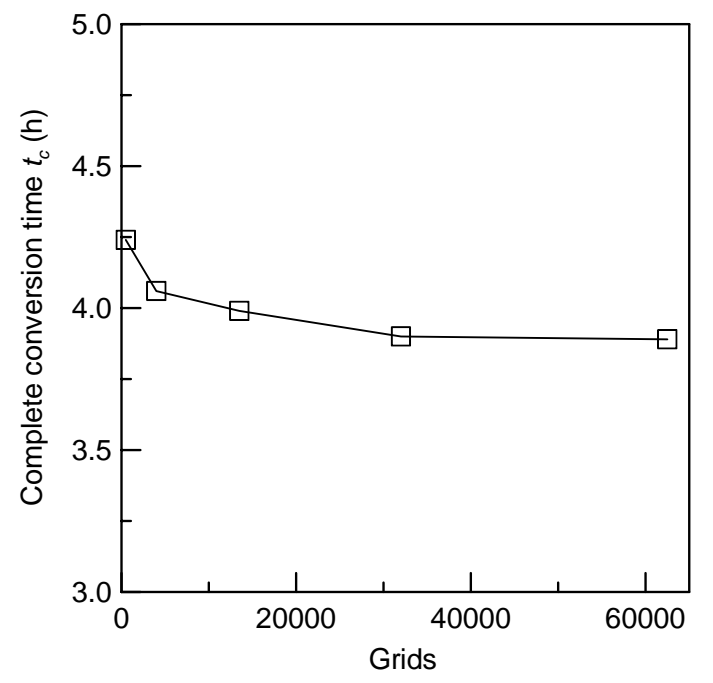

Fig. 3. Effect of grids on the simulated complete conversion time $t_{\mathrm{c}}$.

represents the ratio of the reacted volume to the particle volume. As for the conversion constant $K$, it is defined as

$K=\frac{2 D C_{0}}{\epsilon r_{0}^{2}}$

where $r_{0}$ is the radius of a spherical particle and $C_{0}$ is the concentration of the reactant $A$ on the particle boundary surface. For the reaction condition $\mathrm{A}+\mathrm{B} \rightarrow \mathrm{AB}$, the proportional coefficient $\epsilon$ is equal to $C_{0}$. The conversion constant $K$ is, thus, a function of $D$ and $r_{0}^{2}$.

\section{Results and discussion}

In this study, the diffusion controlled solid-state reaction in a spherical particle with $r=r_{0}$ (particle radius) is simulated. According to the stoichiometric coefficients involved in the reaction $\mathrm{A}+\mathrm{B} \rightarrow \mathrm{AB}$, the concentration specified on the particle boundary for $\mathrm{A}$ is $C_{\mathrm{A}}=C_{0}$. The initial concentration of $\mathrm{B}$ inside the particle has the same value as $C_{0}$. Thus, the maximum concentration of $\mathrm{AB}$ produced in the particle is $C_{\mathrm{AB}}=C_{0}$. According to the conversion constant $K$ defined in Eq. ((9)), different ratios of the diffusivity $D$ to $r_{0}^{2}$ are chosen to render several values of $K$ for simulating the diffusion of $A$ into the spherical particles and its reaction with the reactant $\mathrm{B}$.

\subsection{Grid-independent test}

The calculations are performed on a personal computer. In this simulated problem, no exact solution is available to confirm the prediction accuracy. For this reason, a series of computations should be performed by continuously refining the mesh to determine the grid-independent solution. In this study the simulated complete conversion time $t_{\mathrm{c}}$ at $K=$ $4 \times 10^{-5} \mathrm{~s}^{-1}$ is used as the benchmark value. Fig. 3 illustrates the effect of grid resolution on $t_{\mathrm{c}}$. The minimum allowable number of grids to obtain the grid-independent $t_{\mathrm{c}}$ is found to exceed 20,000. A typical analysis conducted in this paper involves 32,000 grids. The resultant $t_{\mathrm{c}}$ is $3.90 \mathrm{~h}$.

\subsection{Concentration distribution}

Fig. 4(a)-(c) present the reaction front propagating towards the particle center at $t=1.0,2.0$, and $3.0 \mathrm{~h}$,

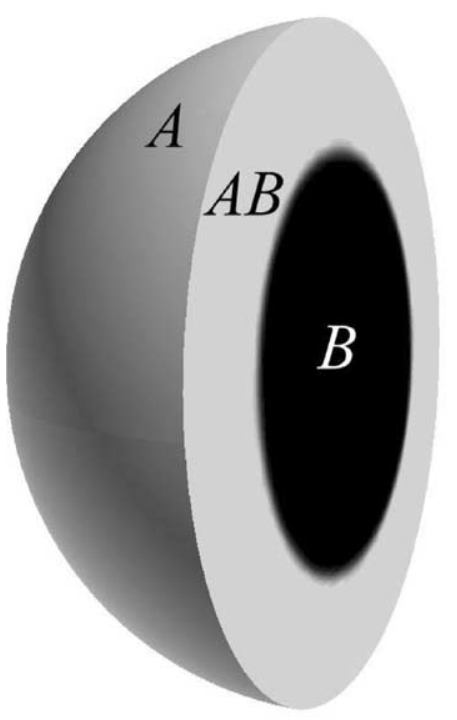

(a)

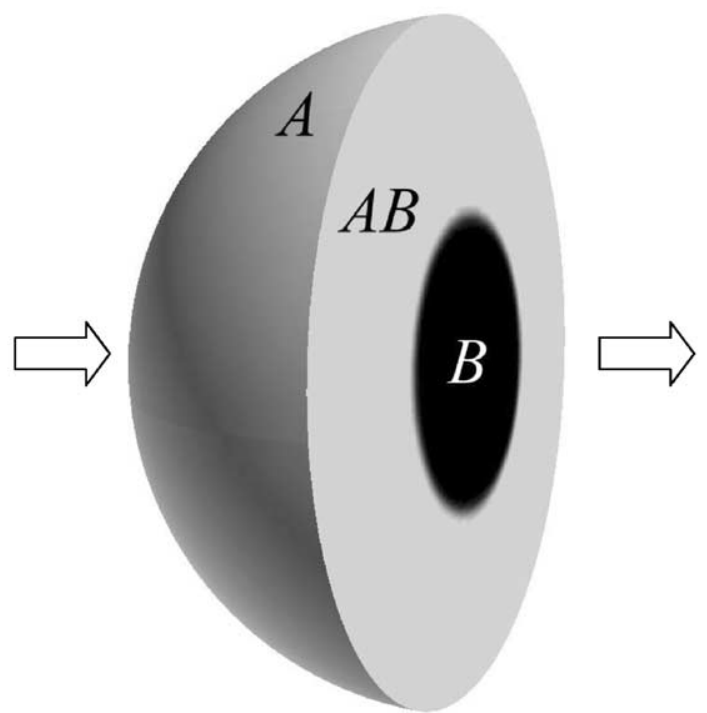

(b)

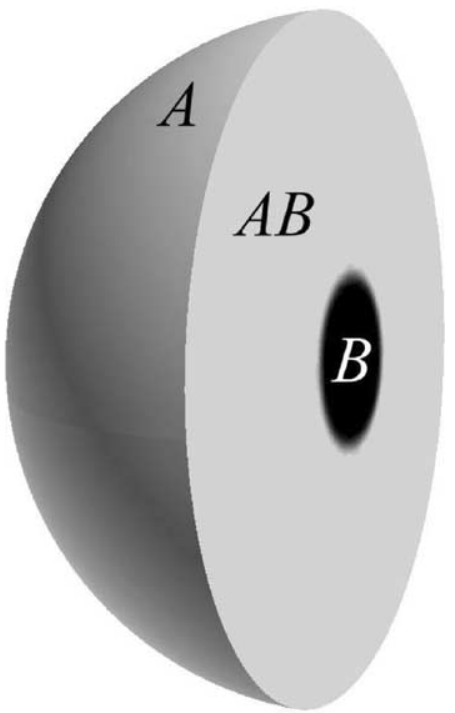

(c)

Fig. 4. An illustration of the reacted zone proceeding towards the particle center at: (a) $t=1.0 \mathrm{~h}$; (b) $t=2.0 \mathrm{~h}$; (c) $t=3.0 \mathrm{~h}$. 


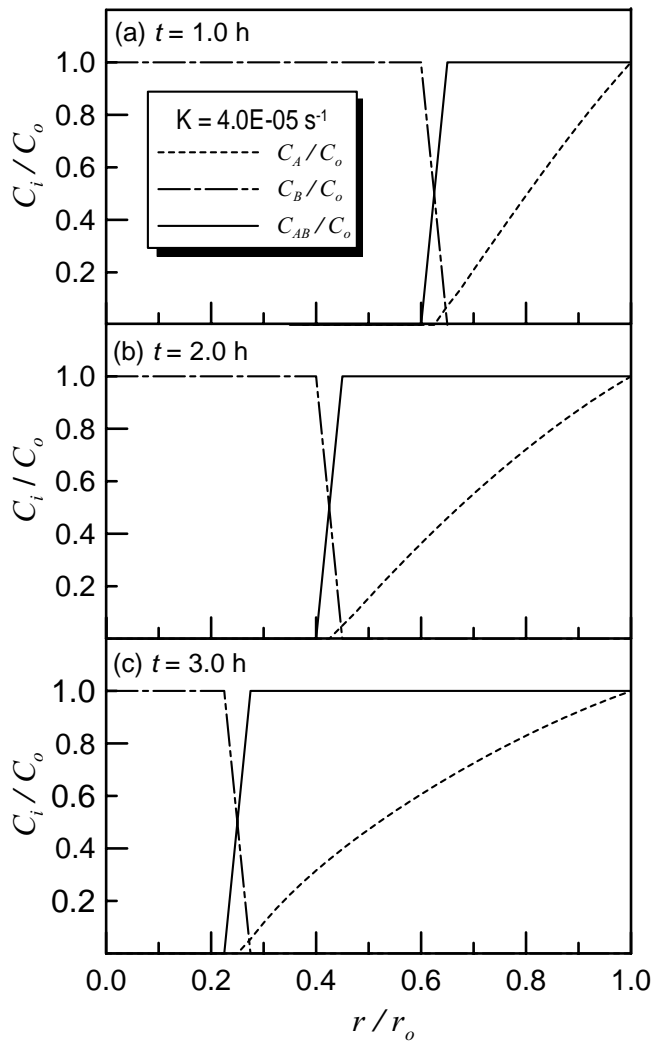

Fig. 5. The simulated normalized concentrations $\left(C_{i} / C_{0}, i=\mathrm{A}, \mathrm{B}\right.$, or $\mathrm{AB})$ for reactants $\mathrm{A}, \mathrm{B}$ and product $\mathrm{AB}$, which are plotted against the normalized radial distance $r / r_{0}$, measured from the center of the spherical particle at: (a) $t=1.0 \mathrm{~h}$, (b) $t=2.0 \mathrm{~h}$, and (c) $t=3.0 \mathrm{~h}$.

respectively, with the conversion constant $K=4 \times 10^{-5} \mathrm{~s}^{-1}$. At $t=1.0 \mathrm{~h}$, the reactant A diffuses into the particle and reacts with $\mathrm{B}$, thus producing a product layer $\mathrm{AB}$. In the course of forming $\mathrm{AB}$, reactant $\mathrm{A}$ keeps diffusing through the product layer $A B$ and reaches the surface of unreacted B. The solid-state reaction occurs instantaneously on the interface and the three-dimensional shrinkage of the reaction interface continues, as shown in Fig.4(b) and (c). For a spherical particle with uniformly specified initial and boundary conditions, it is seen that the shape of the unreacted core remains spherical.

The normalized concentrations $\left(C_{i} / C_{0}, i=\mathrm{A}, \mathrm{B}\right.$, or $\left.\mathrm{AB}\right)$ of reactants $\mathrm{A}, \mathrm{B}$ and product $\mathrm{AB}$ against the normalized radial distance $r / r_{0}$, measured from the center of the spherical particle, at 1.0, 2.0, and 3.0 h are illustrated in Fig. 5. It is seen from these figures that the reactant $\mathrm{A}$ diffuses within the product layer. The concentration $C_{\mathrm{A}}$ decreases gradually from the outer surface of the particle towards the interface between the product layer $\mathrm{AB}$ and the unreacted zone $\mathrm{B}$. This concentration gradient enables $\mathrm{A}$ to diffuse into the product layer. The concentration of $\mathrm{B}\left(C_{\mathrm{B}}\right)$ remains unchanged in the unreacted zone and is, however, zero in the product layer. In between $\mathrm{AB}$ and $\mathrm{B}, \mathrm{B}$ has a residual value since the reaction has not yet completed. The concentration of $\mathrm{AB}\left(C_{\mathrm{AB}}\right)$ is

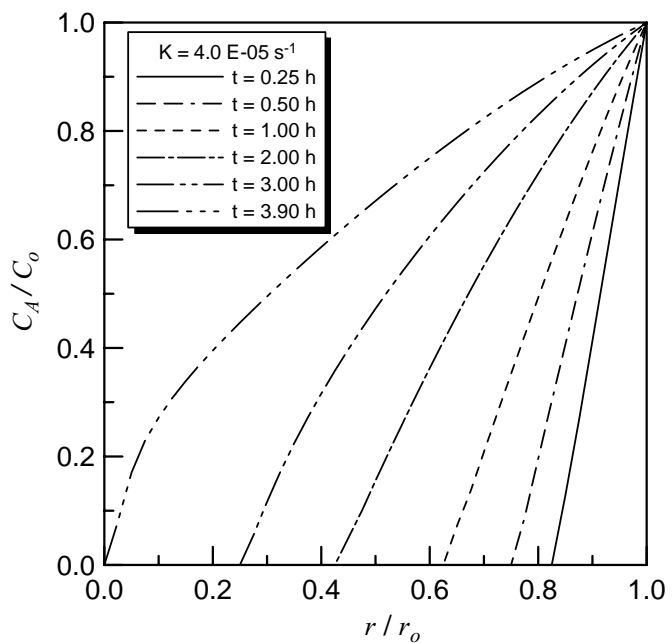

Fig. 6. The simulated normalized concentrations for A against the normalized radial distances $r / r_{0}$, which are measured from the center of the spherical particle, at different periods of time.

equal to that of the reacted $\mathrm{B}\left(C_{\mathrm{B}}\right)$. In addition, Fig. 5(a)-(c) shows that the thickness of $\mathrm{AB}$ increases with time.

In Fig. 6, the normalized concentrations for $\mathrm{A}$ at six different conversion periods are obtained at $K=4 \times 10^{-5} \mathrm{~s}^{-1}$. It can be observed from this figure that the concentration gradient of A becomes smaller with time. This implies that it is comparatively difficult for A to diffuse into the thicker product layer as time keeps going.

\subsection{Diffusion rate}

The normalized diffusion rate $Q / Q_{0}$ is presented as a function of the normalized radial distance to the particle center $r / r_{0}$ in Fig. 7. Here, $Q$ shown below represents the

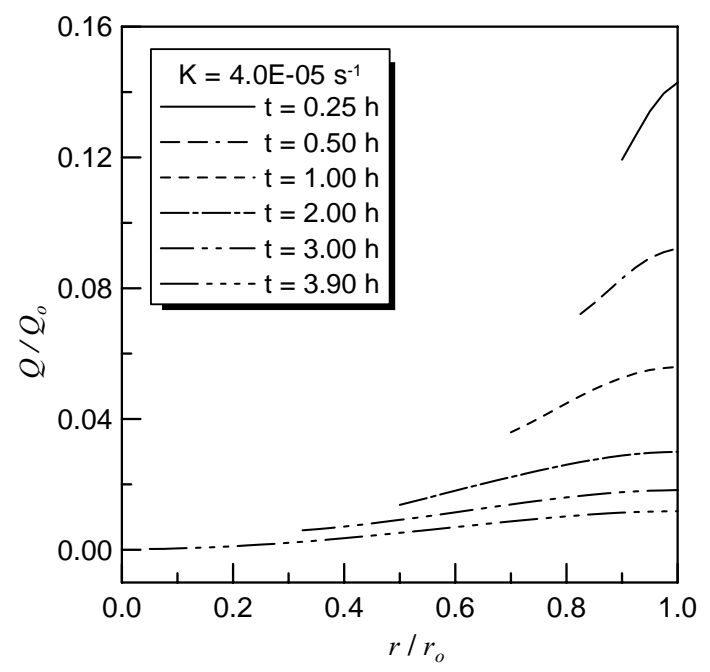

Fig. 7. The simulated normalized diffusion rates $Q / Q_{0}$, which are e0xpressed as a function of the normalized radial distance $r / r_{0}$ to the particle center, for $\mathrm{A}$ at different periods of time. 


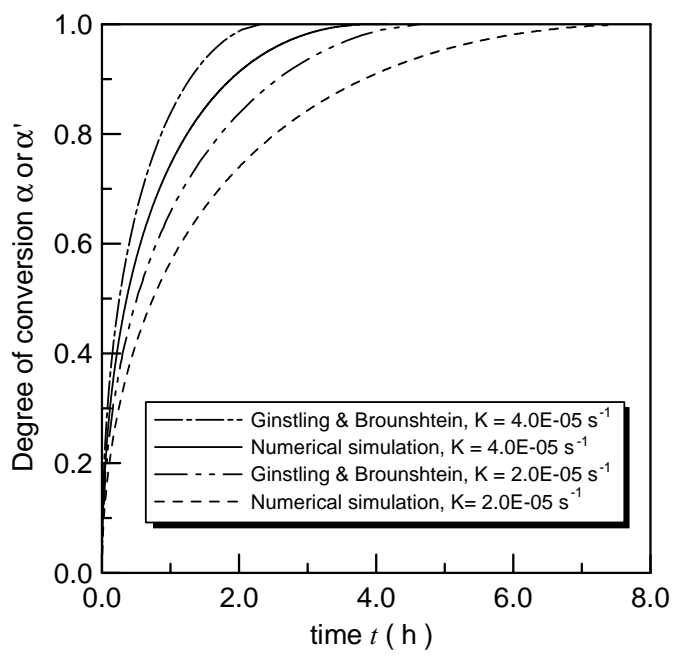

Fig. 8. A comparison of simulated and Ginstling and Brounshtein's kinetic curves.

diffusion rate of $\mathrm{A}$ due to the molecular diffusion through a spherical cross section (with a radius of $r$ ) at $t$

$Q=4 \pi r^{2} D \frac{\Delta C_{\mathrm{A}}}{\Delta r}$

where $\Delta C_{\mathrm{A}}$ is the concentration difference for $\mathrm{A}$ between two ends that are distanced with a length of $\Delta r$. The quantity $Q_{0}$ represents the initial diffusion rate of A through the outer surface of the spherical particle, defined as

$Q_{0}=4 \pi r_{0}^{2} D \frac{C_{0}}{\Delta r}$

It is worthy to note that the diffusion rates for A obtained at different $t$ are all gradually reduced from the outer surface of the particle to the front of the product layer. This enlightens that a net gain of the diffusing reactant $\mathrm{A}$ is seen in the product layer as time marches. The diffusing process in each product layer is, thus, unsteady. It is also noticed that in the direction towards the particle center, the diffusion rate becomes smoothly varying. The implication is that the investigated diffusing system has almost reached a steady state at the end of the conversion. Also note that the diffusion rate at any spherical cross section within the product layer decreases as the conversion proceeds. Up to this point, the diffusion of $\mathrm{A}$ into the product layer can be reduced. In addition, the step-up rate of the degree of conversion is slowed down.

\subsection{Kinetic curves}

The kinetic curves (degree of conversion ( $\alpha$ or $\alpha^{\prime}$ ) versus time $(t))$ at $K=4 \times 10^{-5}$ and $2 \times 10^{-5} \mathrm{~s}^{-1}$ are plotted in Fig. 8. This figure shows that the kinetic curves have the parabolic form. The slopes of the conversion are seen to gradually decrease when the conversion is nearly complete. The explanation for this is due to the decreasing diffusion rate of $\mathrm{A}$ when the thickness of the product layer is in-

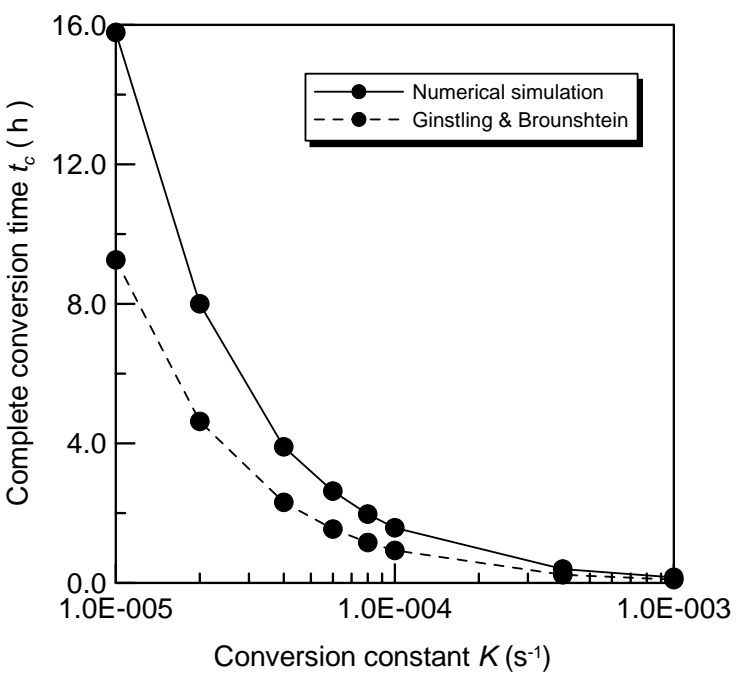

Fig. 9. A comparison of simulated $t_{\mathrm{c}}$ with that of the Ginstling and Brounshtein, which is obtained at different conversion constants $K$.

creased. Moreover, Fig. 8 shows a comparison between the results of simulation $(\alpha-t)$ and Ginstling and Brounshtein's model $\left(\alpha^{\prime}-t\right)$. For the case with $K=4 \times 10^{-5} \mathrm{~s}^{-1}$, the calculated $t_{\mathrm{c}}$ is $3.90 \mathrm{~h}$, while $2.31 \mathrm{~h}$ using the Ginstling and Brounshtein's model. As compared with the numerically simulated $t_{\mathrm{c}}(7.79 \mathrm{~h})$, the Ginstling and Brounshtein's model needs only $4.63 \mathrm{~h}$ for the case with $K=2 \times 10^{-5} \mathrm{~s}^{-1}$. It appears that our simulated result agrees with the Ginstling and Brounshtein's data either in the very beginning of reaction process or for a case considered at a larger conversion constant $K$.

The simulated $t_{\mathrm{c}}$ versus $K$ is also compared with that by Ginstling and Brounshtein's model in Fig. 9. Our simulated result has a good agreement with the Ginstling and Brounshtein's data when the conversion constant $K$ is greater. A possible reason is due to the steady diffusion assumption made by Ginstling and Brounshtein in the reacted zone. Therefore, from the results shown in Figs. 8 and 9, their model is valid only in the initial period of the conversion and for a diffusion system which can quickly reach the steady state.

As shown in Fig. 10, the kinetic curves are obtained at different $K$. According to Eq. (9), the conversion constant $K$ is proportional to the ratio of diffusivity $(D)$ to the square of particle radius $\left(r_{0}^{2}\right)$. In other words, for the diffusion-controlled solid-state reaction in spherical particles, the same kinetic characteristic can be obtained as long as $D / r_{0}^{2}$ remains at a fixed value. With this recognition, the key value for evaluating the conversion kinetics of the diffusion-controlled solid-state reaction in spherical particles is $D / r_{0}^{2}$ or $K$. In Fig. 10, it is obvious that the degree of conversion is higher for the case with a larger value of $K$. Useful information for evaluating the effect of $K$ on the degree of conversion $(\alpha)$ versus time is, thus, obtained. 


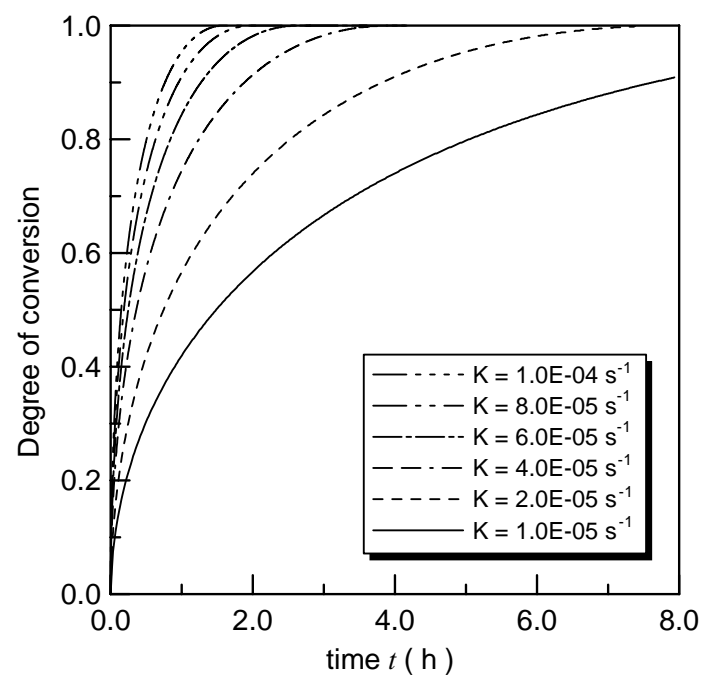

Fig. 10. The degree of conversion against time for the cases obtained at different conversion constants $K$.

\section{Conclusions}

A numerical model for simulating the diffusion-controlled solid-state reaction in spherical particles is developed. This model can accurately predict the characteristics of the conversion system and is, thus, useful in determining the degree of conversion versus time. Moreover, the simulated result clearly shows the propagation of product layer towards the particle center. The unreacted core is seen to remain spherical for a spherical particle with uniform initial and boundary conditions. The computed concentrations for reactants and products can extend our knowledge about the diffusion process of the reactant and the growth history of the product layer. The decreasing concentration gradient of the reactant reveals the difficulty for a reactant to diffuse into the product layer. The diffusion rates of reactant obtained at different spherical cross sections in the reacted layer reveal that the diffusion rates of the reactant are all gradually reduced from the outer surface to the interior. This implies that the diffusing process in each product layer is unsteady. It is, therefore, expected to have a net gain of the reactant $\mathrm{A}$ in the product layer as time goes by. However, the diffusion rate of the reactant on any cross section decreases as the conversion proceeds. An inadequate supplement of the diffusing reactant to the product layer is known to result in a decreased conversion. For the diffusion-controlled solid-state reaction in spherical particles, the key value that can affect the conversion time is $K$ (the ratio of diffusivity to the square of particle radius). Spherical particles with a larger value of $K$ will cause the degree of conversion to increase. Furthermore, the same value of $K$ (or $\left.D / r_{0}^{2}\right)$ will result in the same kinetics of the diffusion-controlled solid-state reaction.

\section{References}

[1] W.H. Sheu, S.F. Tsai, T.P. Chiang, Numer. Heat Transfer, Part A: Applic. 35 (1999) 797.

[2] W. Jander, Z. Anorg. Allg. Chem. 163 (1927) 1.

[3] C. Wagner, Trans. AIME 192 (1952) 214.

[4] M. Avrami, J. Chem. Phys. 7 (1939) 1103.

[5] B.V. Erofe'ev, Comput. Rend. Acad. Sci. URSS 52 (1946) 511.

[6] W.A. Johnson, R.F. Mehl, Trans. AIME 135 (1939) 416.

[7] W.D. Kingery, H.K. Bowen, D.R. Uhlmann, Introduction to Ceramics, Wiley, New York, 1967, p. 420.

[8] A.M. Ginstling, B.I. Brounshtein, J. Appl. Chem. USSR 23 (1950) 1327.

[9] R.E. Carter, J. Chem. Phys. 34 (1961) 2010.

[10] J.F. Thompson, Lecture Series Von Karman Institute, 1978.

[11] J.F. Thompson, Z.U. Warsi, C.W. Mastin, Numerical Grid Generation, 1985.

[12] D.B. Spalding, Int. J. Numer. Methods Eng. 4 (1972) 551.

[13] IBM and Dassault Systems, CATIA V5, 2002.

[14] ICEM CFD Engineering, ICEM-HEXA V4.2, 2002.

[15] AEA Technology, CFX-4.4, 2002.

[16] Intelligent Light, Fieldview V8, 2002. 